\title{
Detecção de Risco de Queda no Ambiente Hospitalar a partir da Informação do Estado do Canal Sem-Fio
}

\author{
Amanda de O. Sabino das Chagas \\ Programa de Pós-Graduação \\ em Engenharia Eletrônica \\ Universidade do Estado \\ do Rio de Janeiro \\ Rio de Janeiro, Brasil \\ chagas.amanda@posgraduacao.uerj.br
}

\author{
Lisandro Lovisolo \\ Programa de Pós-Graduação \\ em Engenharia Eletrônica \\ Universidade do Estado \\ do Rio de Janeiro \\ Rio de Janeiro, Brasil \\ lisandro@uerj.br
}

\author{
Michel P. Tcheou \\ Programa de Pós-Graduação \\ em Engenharia Eletrônica \\ Universidade do Estado \\ do Rio de Janeiro \\ Rio de Janeiro, Brasil \\ mtcheou@uerj.br
}

\begin{abstract}
Resumo - A Informação do Estado do Canal (CSI - Channel State Information) reflete o canal/ambiente de propagação: amplitudes e fases das subportadoras OFDM (Orthogonal Frequency Division Multiplex). Neste trabalho, empregamos a CSI de redes sem-fio IEEE 802.11 para detecção de risco de queda em ambientes hospitalares. Aprende-se o estado normal de CSI e assume-se que alterações no ambiente de propagação sem fio que estão potencialmente relacionadas a riscos de quedas. Descrevemos três detectores usando esse princípio que empregam kNN (K-Nearest Neighbor), PCA (Principal Component Analysis) e Autoencoder. A partir de limiares da Curva ROC, escolhe-se um ponto de corte operacional para o qual obtêm-se acurácias iguais a $88,33 \%, 79,08 \%$, e $82,42 \%$, para os detectores empregando kNN, PCA e Autoencoder, respectivamente.
\end{abstract}

Index Terms - Detecção de queda, classificadores, detecção de anomalias.

\section{INTRODUÇÃO}

Com a demanda crescente por aplicativos de segurança e saúde eletrônicas, a detecção de atividade humana prescindindo de dispositivos específicos (similares a peças de roupa, relógios, pulseiras) ou configuração de câmeras atrai cada vez mais interesse [1]. A grande disponibilidade de redes locais sem fio em praticamente todos os ambientes permite empregá-las para detectar e estimar a ocupação e a movimentação em ambientes internos [2]-[4].

Neste trabalho, avaliamos a possibilidade de detecção da ocupação de ambientes sem uso de câmeras usando a Informação do Estado do Canal (CSI - Channel State Information). A CSI está disponível em diferentes protocolos de redes, especialmente em redes locais sem fio (WLAN - Wireless Local Area Network) que utilizam a Multiplexação por Divisão de Frequências Ortogonais (OFDM - Orthogonal Frequency Division Multiplex) [5], [6]. Em sistemas OFDM, divide-se a banda em subportadoras ortogonais adjacentes, conferindo capacidade de transmissão paralela. O OFDM é especialmente adequado para transmissão de dados em taxas elevadas em ambientes dispersivos com múltiplos retardos [7].

O presente trabalho foi realizado com apoio da Coordenação de Aperfeiçoamento de Pessoal de Nível Superior - Brasil (CAPES) Código de Financiamento 001.
A CSI é uma estimativa da resposta do canal em bandas estreitas - nas subportadoras [8]. Transmitindo símbolos conhecidos em cada subportadora, a partir do símbolo recebido, estima-se o deslocamento de fase e a atenuação, o estado do canal sem fio, em cada subportadora. A CSI detalha as condições de propagação entre transmissor e receptor, refletindo efeitos de espalhamento, desvanecimento e decaimento que podem afetar o enlace. A CSI está disponível na camada PHY (physical/física) refinada do modelo IEEE 802.11 [9], [10].

Pessoas influenciam o ambiente de propagação. Consequentemente, a CSI deve sofrer alterações em função das pessoas e suas posições no ambiente. A hipótese é que mudanças no ambiente, como pessoas de pé ou andando, produzem mudanças no ambiente de rádio-propagação e como efeito mudanças na CSI. Consequentemente, alterações no ambiente de propagação podem derivar da ocupação do ambiente. Este trabalho tem como objetivo implementar um detector de risco de quedas não-intrusivo em ambiente hospitalar a partir da CSI em sistemas dispositivos WiFi/IEEE 802.11.

Atualmente, redes Wi-Fi (IEEE 802.11) estão disponíveis em praticamente todos os locais. Assim, a CSI pode ser uma forma eficiente de realizar a detecção de movimentos em enfermarias e sinalizar possíveis riscos de queda. Ao mesmo tempo, respeitam-se as iniciativas de proibição de filmagem e do uso de câmeras em enfermarias por questões de privacidade de pacientes e sigilo profissional [11], [12].

Há alguns trabalhos com enfoque similar: uso de rede Wi-Fi e equipamentos simples, sem o requisito de hardware especializado para rastreamento de pessoas. Os autores de [13] apresentam um radar passivo WiFi em tempo real, e, os de [14], promovem a detecção de atividades e movimentos humanos por meio de sinais sem fio para assistência médica. Outro exemplo, é o Widar2.0 [15] que visa a localização interna através da CSI. Em [16], visa-se detectar a presença humana e classificá-la extraindo o espalhamento Doppler do sinal refletido, o que exige um processamento bastante mais sofisticado, característico das técnicas de CPL (Coherent Passive Localization). Em [17], é apre- 
sentado o uso dessa ferramenta para reconhecimento de atividades, detecção de respiração e contagem de pessoas. Em [2], faz-se uso da CSI para detecção de entrada e saída de pessoas em um ambiente (interno) obtendo acurácia de 85\%. Em [1], são aplicadas Redes Neurais Profundas (DNN -Deep Neural Networks), SVM (Support Vector Machine), DT (Decision Trees) e GMM (Gaussian Mixture Models) para o monitoramento de atividades humanas como ausência, presença e sonolência em ambiente de trabalho a partir de parâmetros de sinais sem fio, obtendo precisão na classificação das atividades próxima a $93 \%$.

Neste trabalho, desenvolve-se um detector de risco de queda em ambiente hospitalar. Objetiva-se fornecer uma ferramenta que seja utilizável em situações nas quais há pouco contingente disponível de pessoas para monitoramento de pacientes, por exemplo, no turno noturno. $\mathrm{O}$ sistema deve indicar se algum paciente se levantou para que os responsáveis pela enfermaria possam atender pessoalmente ao local sinalizado, inspecionar e tomar as ações necessárias. Consequentemente, o sistema deve aprender o que representa um ambiente desprovido de riscos de queda na CSI. Para isso usamos diferentes abordagens de aprendizado de máquina, obtendo modelos que descrevam e permitam reconhecer se a CSI do ambiente representa a classe/situação sem risco somente. No caso de um paciente se levantar, esperam-se as alterações na CSI. Tais alterações possuem rebatimento nas saídas dos modelos, permitindo sinalizar o risco de queda. Isto é, o risco de queda é detectado como uma anomalia do estado do canal correspondente a sem risco de queda.

Testamos tal abordagem usando as técnicas de aprendizado de máquina kNN, PCA e Autoencoder. A escolha por esses algoritmos têm motivações distintas. O kNN é uma técnica simples, mas com capacidade de identificar variações e detectar diferenças entre os padrões originais de uso amplo e extenso. O PCA permite representar comprimidamente os dados de entrada (redução de dimensionalidade) usando uma metodologia linear e podemos assim comparar os padrões num espaço de menor dimensão. Já o Autoencoder é capaz de aprender a representação compacta a partir dos dados originais de forma não linear.

A Seção II discorre brevemente sobre CSI e descreve como o sistema implementado captura essa informação para a detecção de risco de queda em ambiente hospitalar. A Seção III descreve como cada um dos três classificadores (baseados em kNN, PCA ou Autoencoder) processam o CSI para detectar a anomalia, isto é, o risco de queda. A Seção IV descreve o processo de coleta dos dados e os resultados dos experimentos são apresentados na Seção V. A Seção VI apresenta as conclusões.

\section{Captura do CSI em Redes IEEE 802.11}

\section{A. CSI Disponível no IEEE 802.11}

O conjunto de padrões IEEE 802.11 descreve diferentes técnicas das camadas de Controle de Acesso ao Meio (MAC - Medium Access Control) e Camada Física (PHY
- Physical Layer) para WLANs. Na camada PHY, encontramos diferentes técnicas de transmissão e codificação como FHSS (Frequency Hopping Spread Spectrum), DSSS (Direct Sequence Spread Spectrum) e OFDM. Os padrões IEEE $802.11 \mathrm{a} / \mathrm{g} / \mathrm{n}$ empregam OFDM. Neste trabalho, consideramos as redes IEEE 802.11n com canais de $20 \mathrm{MHz}$. A detecção de risco de queda é realizada por meio das amostras CSI de subportadoras L-LTF (nonHT Long Training Field) e HT-LTF (High-Throughput Long Training Field), que são campos nos preâmbulos do padrão IEEE 802.11n e contêm informações sobre as subportadoras e estimativas do canal.

\section{B. Sobre a CSI}

No domínio da frequência, podemos modelar a transmissão usando OFDM através de um canal via

$$
\mathbf{y}=\mathbf{H x}+\mathbf{n}
$$

na qual, $\mathbf{x}$ representa o símbolo OFDM transmitido (um vetor complexo), y é o símbolo recebido, $\mathbf{H}$ é a matriz de canal, e $\mathbf{n}$ é o ruído.

A resposta em cada subportadora $i$ é

$$
h_{i i}=h_{i}=\left|h_{i}\right| e^{j \sin \left(\angle h_{i}\right)},
$$

onde a $\left|h_{i}\right|$ representa a amplitude e $\angle h_{i}$ representa a fase da $i$-ésima subportadora, que compõe a CSI fornecida pelo IEEE 802.11.

\section{Geração, Captura e Tratamento da CSI}

Para monitorar o ambiente e avaliar mudanças da CSI, empregam-se módulos ESP32 [18] - microcontroladores de baixo custo e baixo consumo de energia projetados visando aplicações IoT (Internet-of-Things). Esses módulos suportam vários protocolos de redes sem fio como $802.11 \mathrm{~b} / \mathrm{g} / \mathrm{n}$ e Bluetooth, entre outros.

Programam-se os módulos para coleta da CSI de rede WiFi. Um módulo ESP32 é programado para realizar transmissões periódicas a um ponto de acesso WiFi, para medição periódica da CSI. Outro, coleta a CSI entre o primeiro módulo e o ponto de acesso, armazena-a e transmite-a para processamento e análise pelo sistema de detecção de quedas. Capturam-se 2 amostras CSI (com 128 subportadoras L-LTF e HT-LTF) por segundo.

A CSI de cada subportadora contém uma parte real e outra imaginária; das quais obtêm-se os módulos e as fases correspondentes a cada subportadora. Porém, a estimativa de fase usando dispositivos de baixo custo é imprecisa (não confiável) devido a imperfeições nos componentes eletrônicos e erros de sincronização [2], [3]. Assim, para a detecção do risco de queda, empregamos somente os módulos das subportadoras, como mostra a Figura 1.

\section{Sistema de DetecçÃo do Risco De QuedA}

A detecção de risco de queda em ambiente hospitalar se torna relevante em horários de pouca movimentação de pessoal. Por exemplo, não há em geral alguém para 


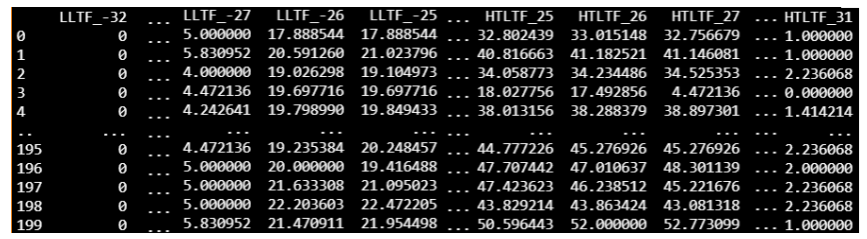

Figura 1. Módulo das Subportadoras OFDM (CSI).

observar se pacientes internados se levantam a noite. O sistema detector deve ser capaz de diferenciar entre a situação/classe "Sem risco de queda" e "Com risco de queda". Desenvolvemos tais sistemas para detecção de queda usando três algoritmos de aprendizado de máquina: kNN, PCA e Autoencoder. Tenta-se reproduzir o cenário alvo realizando testes em ambiente pouco movimentado. Nele, são simuladas as duas situações (classes) para avaliar se mudanças no ambiente podem ocasionar mudanças no canal sem fio produzindo alterações na CSI. E, assim detectar situações de risco de queda a partir da detecção de anomalias na CSI.

O detector de risco de queda (considerando qualquer um dos três algoritmos) é treinado para aprender a situação "Sem risco de queda", essa reflete a situação normal do ambiente da enfermaria. Repara-se que ela pode ser aprendida rapidamente sem expor ninguém a riscos ao dispor no ambiente dispositivos de transmissão e coleta (Seção II-C). Além disso, parece ser inviável obter todos os padrões possíveis de CSI de situações de risco de queda. Assim, aborda-se o problema treinando os sistema somente com amostras "Sem risco de queda" de forma que circunstâncias diferentes das aprendidas sejam consideradas situações anormais. A associação do risco de queda à detecção de um anomalia permite generalizar e detectar situações de risco de queda, possivelmente, não vislumbradas, já que as pessoas variam em muito fisicamente e em seus movimentos.

\section{A. $k N N$}

$\mathrm{O}$ algoritmo kNN ( $\mathrm{K}$ vizinhos mais próximos) é considerado um dos métodos de classificação mais simples, mas ainda assim efetivo [19]. Os dados são classificados a partir de $k$ exemplos mais próximos dentre um conjunto de padrões aprendidos como representativos da classe. Isso exige definir uma medida de distância entre padrões e estabelecer o número de exemplos $k$.

A Figura 2 apresenta o diagrama correspondente à aplicação do kNN neste trabalho. Aprende-se somente a partir de amostras de "Sem risco de queda". Durante a aplicação do detector de risco de queda, computa-se a distância entre a amostra de entrada, o CSI, e os $k$ vizinhos aprendidos (somente amostras "Sem risco de queda") empregamos a distância Euclidiana. Calcula-se a distância média correspondente (ou equivalentemente total). Se a distância média está abaixo de um limiar então a entrada é classificada como "Sem risco de queda". Caso contrário, a entrada é classificada como "Com risco de queda". Isto é, para amostras da classe "Com risco de queda", espera-se que a distância média aos padrões aprendidos seja maior já que eles são aprendidos com a situação (classe) "Sem risco de queda".

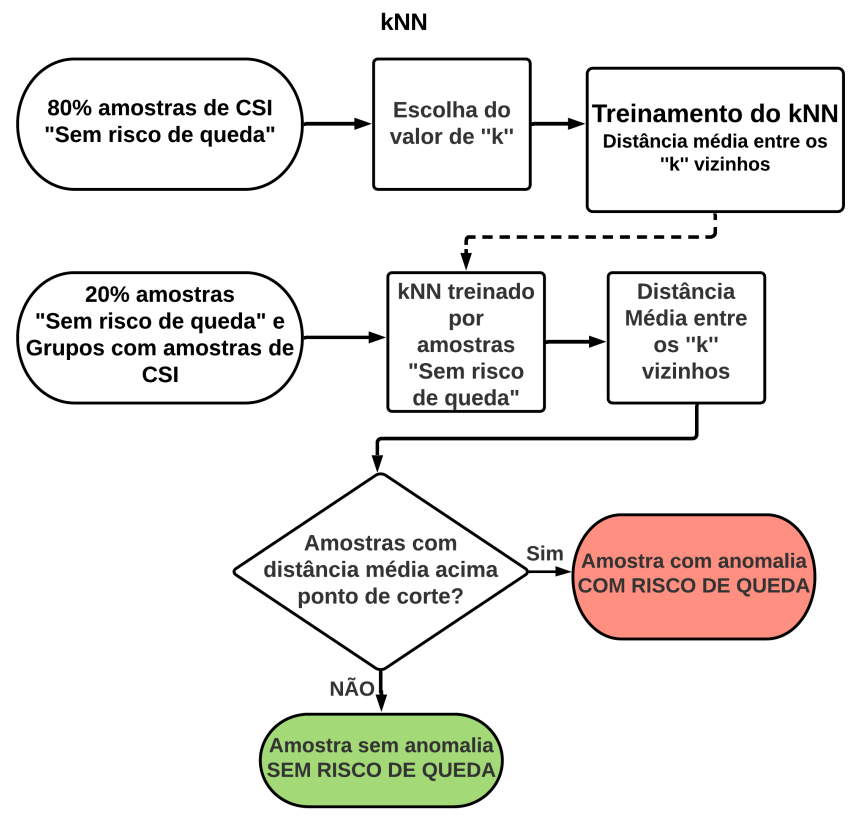

Figura 2. Detector de risco de quedas baseado em kNN.

\section{B. Principal Component Analysis - PCA}

PCA é uma técnica para redução de dimensionalidade [19], [20]. Projeta-se o vetor de entrada num espaço alternativo, cada coordenada no novo espaço corresponde a combinações lineares de coordenadas do vetor de entrada. A primeira coordenada de saída concentra a maior parte da energia dos vetores esperados na entrada. A segunda saída corresponde a direção ortogonal à da primeira; e, assim, sucessivamente. Cada uma delas é dita uma componente principal (CP). Para as CPs do conjunto de treinamento, "Sem risco de queda", obtém-se a variância explicada (a proporção da energia em cada CP) para determinar o número $P$ de CPs a empregar para reduzir a dimensionalidade do CSI.

Para detectar anomalia em uma amostra de CSI, reconstrói-se a amostra a partir das $P$ CPs e calculase o erro de reconstrução - o erro médio quadrático (MSE - Mean Squared Error) entre a CSI e sua versão reconstruída. O diagrama que explica esta estratégia é apresentado na Figura 3. Estratégia similar para detecção de anomalias pode ser vista em [21]. Como as $P$ CPs são obtidas para a classe "Sem risco de queda", esperase que amostras com alto erro de reconstrução indiquem anomalias e, portanto, risco de queda. Assim, a aplicação de um limiar de erro de reconstrução aceitável permite 
diferenciar entre situações normais ("Sem risco de queda") e de anômalas ("com risco de queda").

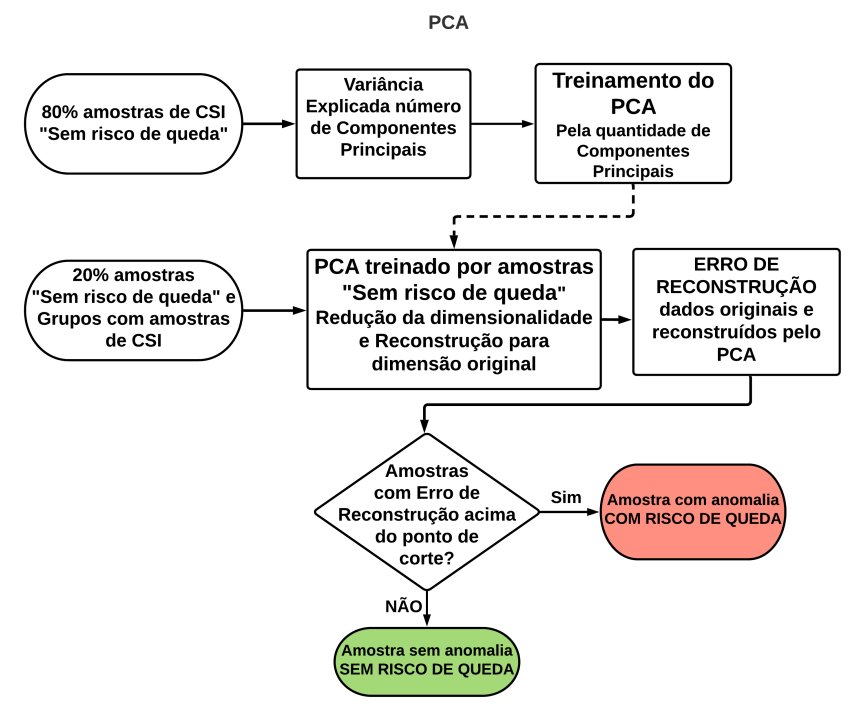

Figura 3. Detector de risco de quedas baseado em PCA.

\section{Autoencoder}

Um Autoencoder é uma rede neural em camadas com capacidade de redução (não linear) da dimensionalidade. Neste estudo, é utilizado um Autoencoder Undercomplete. Ele é composto por codificador e decodificador tais que o treinamento opera de forma que as saídas do decodificador sejam similares às entradas do codificador. Entre os dois, obtém-se uma representação compacta numa "camada escondida" do padrão apresentado na entrada.

Usamos o Autoenconder para detecção de anomalias ("Com risco de queda") tal qual como o PCA. O Autoenconder é treinado para codificar bem amostras de CSI advindas da situação "Sem risco de queda". Essa estratégia é ilustrada na Figura 4. A abordagem é análoga à empregada com PCA. Porém, devido ao processamento não-linear realizado pelo Autoenconder, espera-se, a princípio, maior capacidade de compactação/redução de dimensionalidade e assim possivelmente um melhor desempenho do detector. Procedimento similar é discutido em [22] para deteç̧ão de anomalias.

\section{Coleta de Dados}

A Figura 5 ilustra as situações consideradas para formar o banco de dados de CSI empregado para desenvolver os detectores anteriormente discutidos. A coleta ocorreu num quarto de $9 \mathrm{~m}^{2}$ de área e mobiliado. A Figura 5 mostra a planta baixa do ambiente com a localização da mobília, dos microcontroladores e do ponto de acesso. O microcontrolador ESP32(1) envia dados usando o protocolo UDP (User Datagram Protocol) para o ponto de acesso. Já o ESP32(2) captura a CSI e a transfere para o computador. As situações simuladas são:

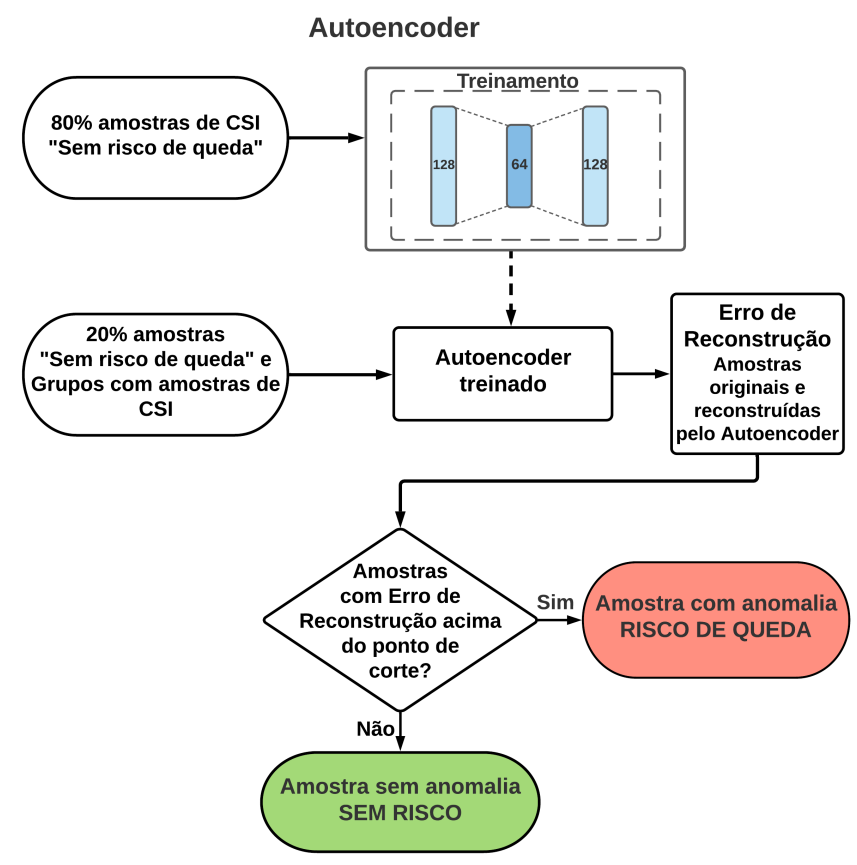

Figura 4. Detector de risco de quedas baseado em Autoencoder.

a) Sem risco de queda: o voluntário permanece deitado na cama colocada no ambiente de testes (um quarto). São coletadas 3000 amostras de CSI nesse estado. Aplicase um hold-out para divisão das amostras para treino $(80 \%$ - 2400 registros) e testes (20\% - 600 registros) do detector de risco de quedas.

b) Com risco de queda: o voluntário levanta da cama e ou caminha, ou corre ou simula uma queda, movimentos que diferem da situação "Sem risco de queda".

As amostras coletadas para análise pelo sistema de detecção de quedas são divididos em três grupos:

Grupo 1 - O voluntário levanta e caminha. Este Grupo possui 200 amostras da classe "Com risco de queda".

Grupo 2 - O voluntário levanta e permanece parado em pé próximo à cama. O Grupo contém 100 amostras de cada classe, "Sem risco de queda" e "Com risco de queda", respectivamente.

Grupo 3 - O voluntário simula uma queda e permanece deitado no chão. Este grupo contém 84 amostras da classe "Sem risco de queda" e 116 amostras da classe "Com risco de queda".

\section{Resultados}

\section{A. Metodologia de Avaliação}

O desempenho da separação dos registros de CSI entre "Sem risco de queda" e "Com risco de queda" são avaliados para os três métodos usando Curvas ROC (Receiver Operating Characteristic Curve). A Curva ROC é obtida variando-se limiares de decisão e registrando a quantidade de registros das classes positiva e negativa [23]. A curva 


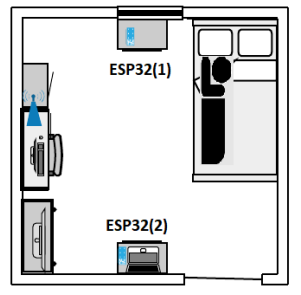

(a) Sem risco de queda

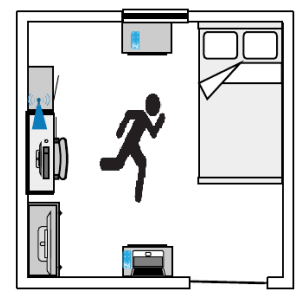

(b) Com risco de queda
Figura 5. Situações simuladas.

ROC relaciona a taxa de verdadeiros positivos TPR (True Positive Rate) ou Sensibilidade com a taxa de falsos positivos FPR (False Positive Rate) ou "1 - Especificidade" e permite visualizar o desempenho de um classificador [24]. Dela, obtém-se a Área sob a Curva AUC (Area Under the Curve) $0 \leq \mathrm{AUC} \leq 1$ que quanto mais próxima a 1 indica melhor desempenho.

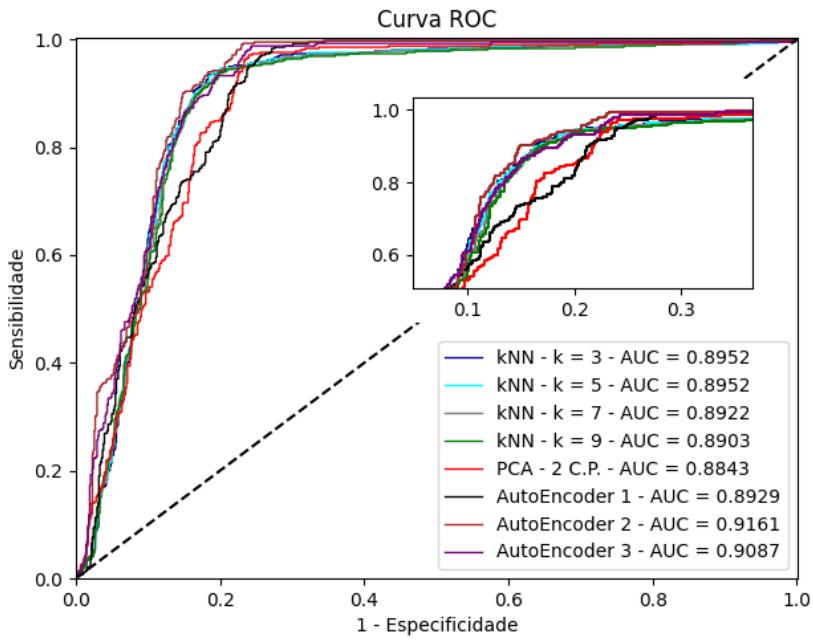

Figura 6. Curva ROC e valores da AUC dos classificadores avaliados. Destaca-e a região em que as curvas mudam de comportamento.

A Figura 6 apresenta as curvas ROC de cada um dos classificadores implementados - os hiper-parâmetros de cada são descritos nas subseções correspondentes a seguir. Cada curva ROC é obtida aplicando os detectores às 600 amostras de teste da classe "Sem risco de queda" e às referentes aos três Grupos da Seção IV. Observamos que a classe "Sem risco de queda" é majoritária e é a classe negativa, enquanto a classe "Com risco de queda" é minoritária, mas é a positiva. O eixo de Sensibilidade na curva ROC indica a capacidade do classificador para identificar corretamente as amostras da classe positiva, "Com risco de queda". Por outro lado, a Especificidade é a capacidade do classificador para identificar corretamente amostras da classe negativa, "Sem risco de queda".

Em geral, busca-se o melhor compromisso entre Sensibilidade e Especificidade, ou seja, define-se o limiar que satisfaça um compromisso entre falsos positivos e falsos negati- vos [23]. Para selecionar o ponto ótimo de corte, podemos usar a Curva TG-ROC (Two-Graph Receiver Operating Characteristic) que apresenta ambas a Sensibilidade e a Especificidade no eixo das abcissas [25]. O ponto de corte é em geral adotado como o limiar de interseção entre as curvas no TG-ROC, ou seja, o limiar para o qual Sensibilidade e Especificidade são aproximadamente iguais. O Ponto de Corte define o funcionamento do classificador. Caso a amostra da CSI analisada produza uma saída (distância ou erro) inferior ao ponto de corte, considera-se que a mesma pertence à classe "Sem risco de queda". Por outro lado, se ela está acima do limiar, considera-se que ela pertence à classe "Com risco de queda", como apresenta a Figura 7.

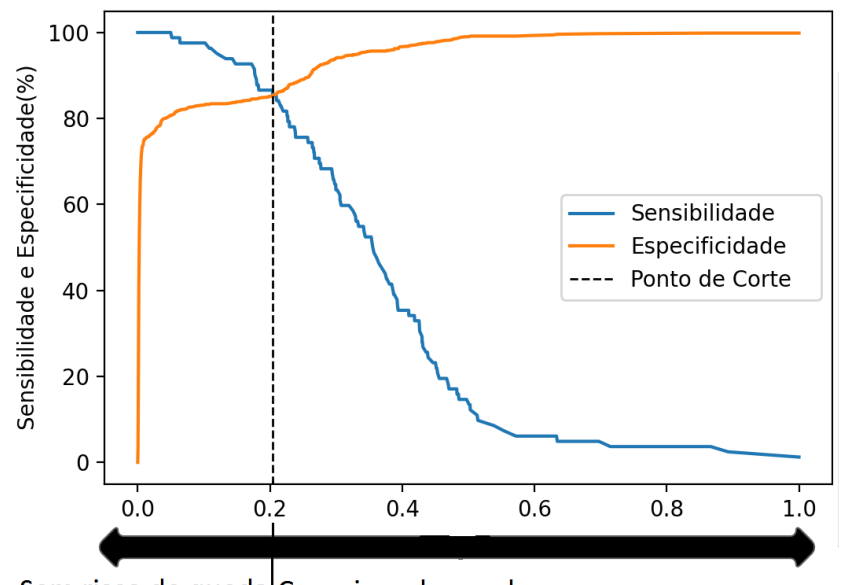

Sem risco de queda|Com risco de queda

Figura 7. Curva TG-ROC para escolha do ponto de corte e classificação das amostras.

\section{B. $k N N$}

Treinam-se quatro classificadores $\mathrm{kNN}$ com $k$ igual a $3,5,7$ e 9 . A configuração com $k$ igual a 3 apresenta melhor desempenho, quando consideramos a Curva ROC da Figura 6, pois obtém AUC maior que as outras. A Figura 8 apresenta os gráficos de Sensibilidade e Especificidade em função do ponto de corte para os diferentes grupos separadamente. Para obtenção dessas curvas, as amostras da classe "Com risco de queda" de cada um dos três grupos são aplicadas separadamente ao classificador. Porém, as amostras da classe "Sem risco de queda" são as mesmas em todos os casos (bem como os classificadores).

A análise por grupos permite observar se há comportamentos distintos em diferentes situações de risco e os efeitos que essas diferenças podem produzir na escolha do ponto de corte. Na Figura 8, o ponto de corte ótimo (seguindo o critério de especificidade e sensibilidade iguais) está indicado em linha tracejada, considerando testes em cada um dos grupos 1, 2 e 3 de risco de queda (Há 600 de registros "Sem risco de queda", enquanto "Com risco de queda" há 200 no grupo 1, há 100 no 2 e há 116 no grupo). A Tabela I apresenta os desempenhos usando esse 
limiar. Alcançam-se taxas de acertos maiores que $89,00 \%$ em todos os grupos.



Figura 8. Desempenho do detector kNN.

Tabela I

Desempenho do DETECTOR KNN NOS PONTOS DE CORTE Ótimos.

\begin{tabular}{|c|c|c|c|}
\hline Grupos & Ponto de Corte & TPR(\%) & TNR(\%) \\
\hline $\mathbf{1}$ & 0,012 & 95,50 & 95,50 \\
\hline $\mathbf{2}$ & 0,026 & 91,00 & 90,28 \\
\hline $\mathbf{3}$ & 0,016 & 89,65 & 89,62 \\
\hline
\end{tabular}

Quando consideramos o grupo 1, obtemos 95,50\% de Sensibilidade e de Especificidade com poucas amostras da classe "Sem risco de queda" classificadas incorretamente, isto é, produzindo falsos positivos. Ao considerarmos o grupo 2, a Sensibilidade é igual a 91,00\% e a Especificidade igual a $90,28 \%$. E para o grupo 3, a Sensibilidade é igual a $89,65 \%$ e a Especificidade igual a $89,62 \%$. Nota-se que os limiares de corte obtidos separadamente para os 3 grupos são bastante próximos.

\section{C. $P C A$}

Para o classificador usando PCA (Seção III-B) avaliouse que 2 CPs são suficientes para acumular uma Variância Explicada igual a 95,00\% para 85,92\% dos dados do conjunto de treinamento (somente casos "Sem risco de queda"). Assim, empregamos o PCA para reduzir os 128 componentes das amostras de CSI para 2 CPs, e subsequentemente, efetuar a reconstrução dos dados (128 componentes) e calcular o erro de reconstrução. A Figura 9 apresenta as sensibilidades e especificidades para os grupos 1, 2 e 3, seguindo a metodologia descrita na subseção V-B. A Tabela II apresenta os desempenhos nos pontos de corte ótimos. Alcançam-se taxas de acerto maiores que 90,00\% nos três grupos.

Quando consideramos o grupo 1, obtemos 96,00\% de Sensibilidade e de Especificidade com poucas amostras da classe "Sem risco de queda" classificadas incorretamente,
Sensibilidade (TPR) e Especifidade (TNR) - PCA

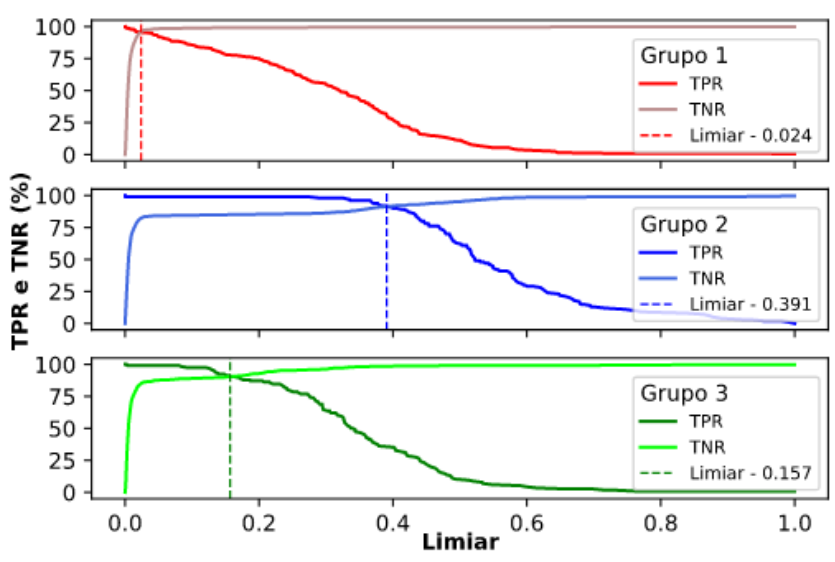

Figura 9. Desempenho do detector PCA.

Tabela II

Desempenho do Detector PCA no ponto de Corte.

\begin{tabular}{|c|c|c|c|}
\hline Grupos & Ponto de Corte & TPR(\%) & TNR(\%) \\
\hline $\mathbf{1}$ & 0,024 & 96,00 & 96,00 \\
\hline $\mathbf{2}$ & 0,391 & 92,00 & 91,28 \\
\hline $\mathbf{3}$ & 0,157 & 90,51 & 90,46 \\
\hline
\end{tabular}

isto é, produzindo falsos positivos. Já ao considerarmos o grupo 2, a Sensibilidade é igual a $92,00 \%$ e a Especificidade igual a 91,28\%. Para o grupo 3, ocorre situação semelhante, com Sensibilidade igual a 90,51\% e Especificidade igual a $90,46 \%$. Nota-se que os limiares de corte obtidos para os 3 grupos são bastante diferentes, porém, vemos também que o ponto de corte obtido para o grupo 1 pode ser aplicado em todos sem grande perda de desempenho.

\section{Autoencoder}

Testamos três configurações de Autoenconder para detector descrito na Seção III-C. O Autoencoder é treinado usando 20 épocas, com tamanho de lote (batch size) igual a 128, com otimizador Adam e Early Stopping (caso não ocorra redução da perda em 2 épocas, o treinamento é interrompido). Foram realizados testes com três modelos de Autoencoder com diferentes quantidades de camadas e neurônios: Modelo 1 com sete camadas de 128-64-3216-32-64-128 neurônios, Modelo 2 com cinco camadas de 128-64-32-64-128 neurônios e Modelo 3 com três camadas de 128-64-128 neurônios. O Modelo 2 apresentou melhor desempenho (maior AUC) sendo analisado aqui.

A Figura 10 apresenta as Sensibilidades e Especificidades para os grupos 1, 2 e 3, seguindo a mesma metodologia acima. A Tabela III apresenta o desempenho no ponto de corte. Alcançam-se taxas de acertos maiores que $89,00 \%$ nos três grupos.

Similarmente ao observado para os detectores de risco de queda baseados em kNN e PCA, também para o baseado em Autoencoder, no grupo 1 observam-se menos falsos 
Sensibilidade (TPR) e Especifidade (TNR) - Autoencoder

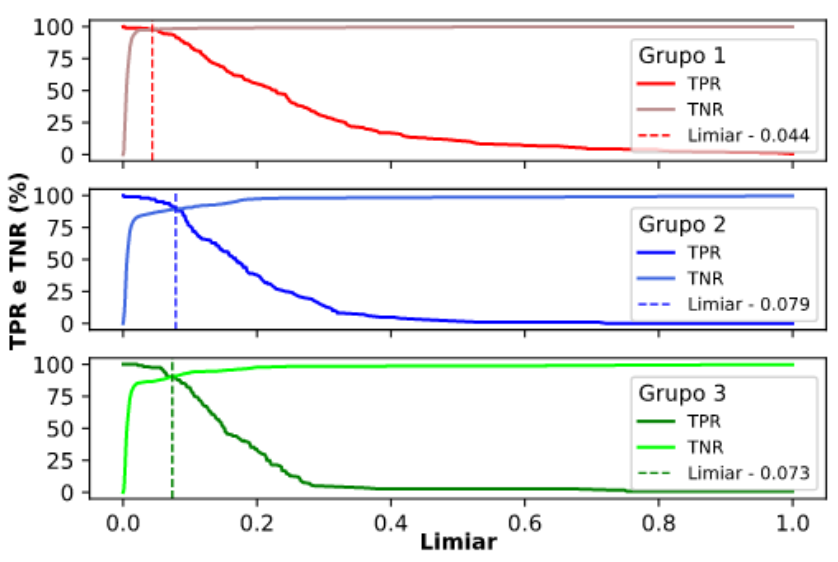

Figura 10. Desempenho do detector Autoencoder.

Tabela III

Desempenho do detector Autoencoder no ponto de Corte.

\begin{tabular}{|c|c|c|c|}
\hline Grupos & Ponto de Corte & TPR(\%) & TNR(\%) \\
\hline $\mathbf{1}$ & 0,044 & 97,50 & 97,50 \\
\hline $\mathbf{2}$ & 0,079 & 90,00 & 89,42 \\
\hline $\mathbf{3}$ & 0,073 & 90,51 & 90,49 \\
\hline
\end{tabular}

positivos que nos grupos 2 e 3. Para o grupo 1, obtém-se a previsão correta de $97,00 \%$ das amostras e o detector $\mathrm{Au}$ toencoder apresenta maiores Sensibilidade e Especificidade que os outros detectores. Já para o grupo 2, obtêm-se Sensibilidade igual a $90,00 \%$ e Especificidade igual a $89,42 \%$. Para o grupo 3, observa-se desempenho semelhante ao do grupo 2, com Sensibilidade igual a 90,51\% e Especificidade igual a $90,49 \%$. Os pontos de corte para cada um dos três grupos são diferentes, porém mais próximos que os obtidos separadamente para o classificador PCA e menos próximos que para os obtidos para o classificador $\mathrm{kNN}$.

\section{E. Análise de Resultados}

Para a avaliação do desempenho dos classificadores no ponto de corte utilizamos a acurácia: o percentual de amostras classificadas corretamente independentemente da classe. A Tabela IV apresenta as acurácias por grupos (o conjunto "Sem risco de queda" é sempre o mesmo) e classificador, aplicando os limiares de corte correspondentes. Como os três grupos possuem quantidades de amostras diferentes, a comparação direta dos valores é inapropriada, mas, como anteriormente, esse não é o objetivo. Em todos os casos, a acurácia é próxima a 90,00\%.

A próxima avaliação é realizada pelo desempenho dos classificadores no ponto de corte, de amostras por classe. É analisada a acurácia considerando o total de amostras por classe, com 784 amostras da classe "Sem risco de queda" e 416 da classe "Com risco de queda" no total de 1200 registros. A classe "Sem risco de queda" é formada por 600 registros de teste "Sem risco de queda" e por 184 amostras
Tabela IV

ACURÁCIA (\%) NOS DIFERENTES GRUPOS DE RISCO DE QUEDA.

\begin{tabular}{|c|c|c|c|}
\hline & \multicolumn{3}{|c|}{ Classificadores } \\
\hline Grupos & kNN & PCA & Autoencoder \\
\hline $\mathbf{1}$ & $95,50 \%$ & $96,13 \%$ & $97,50 \%$ \\
\hline $\mathbf{2}$ & $90,38 \%$ & $91,38 \%$ & $89,50 \%$ \\
\hline $\mathbf{3}$ & $89,63 \%$ & $90,50 \%$ & $90,50 \%$ \\
\hline
\end{tabular}

dos Grupos 2 e 3 em períodos que o voluntário permaneceu deitado na coleta da CSI. A classe "Com risco de queda" é composta por registros dos Grupos 1, 2 e 3, em momentos que o voluntário movimentou-se pelo ambiente de testes, com total de 416 registros. A Tabela V mostra que os classificadores apresentam bom desempenho apesar do número de amostras por classe não estar balanceado, com resultados acima de 79,08\% no $\mathrm{PCA}, \mathrm{kNN}$ com acurácia de $88,33 \%$ e Autoencoder $82,42 \%$.

Tabela V

ACURÁCia (\%) DOS Classificadores testados.

\begin{tabular}{|c|c|c|c|}
\hline & \multicolumn{3}{|c|}{ Classificador } \\
\hline & kNN & PCA & Autoencoder \\
\hline Ponto de Corte & 0,038 & 0,208 & 0,097 \\
\hline Acurácia & $88,33 \%$ & $79,08 \%$ & $82,42 \%$ \\
\hline
\end{tabular}

a) Sobre o Ajuste do Ponto de Corte: A situação "Com risco de queda" não identificada corretamente deve ser evitada pois uma queda ou acidente sem rápida assistência pode ser decisiva para o paciente. Por outro lado, situações "Sem risco de queda" que produzem falsos positivos podem acionar a equipe de enfermagem desnecessariamente. Assim, o ponto de corte pode ser ajustado para tornar os classificadores mais flexíveis ou mais conservadores. Um classificador mais flexível tem Sensibilidade maior que a Especificidade, produzindo mais falsos positivos. Um classificador mais conservador tem Sensibilidade menor que a Especificidade, produzindo menos falsos positivos mas podendo prejudicar a deteç̧ão da situação "Com risco de queda". Em uma enfermaria, uma alternativa é empregar diferentes pontos de corte conforme a movimentação na enfermaria. Por exemplo, no período diurno, com mais funcionários e o movimento de pacientes e visitas, empregar-se-ia um limiar de corte conservador. Já no período noturno, com menor movimento, o uso de um limiar de corte que produzisse uma classificação mais flexível permitiria a rápida ação da equipe de enfermagem.

\section{Conclusões}

Este trabalho apresentou como empregar classificadores de classe única para detectar risco de queda em enfermarias a partir da informação de estado do canal (CSI) de redes sem fio. Devido à grande variabilidade de situações de risco de queda, os detectores são treinados para aprender partir da situação "Sem risco de queda". Assim, a situação se deseja detectar "Com risco de queda" corresponde a uma 
anomalia da situação "Sem risco de queda". Testou-se tal abordagem usando detectores de anomalias baseados em kNN, PCA e Autoencoder. As anomalias são identificadas ou pela distância média da CSI de entrada aos padrões de CSI armazenados, no caso do $\mathrm{kNN}$, ou pelo erro de reconstrução da CSI de entrada nos casos de PCA e Autoencoder. Os três classificadores apresentaram altas taxas de acerto. Verificou-se que os detectores baseados em PCA e Autoencoder apresentam menores proporções de falsos positivos e taxas de acerto levemente superiores. Como observado nos resultados, os pontos de corte de cada Grupo apresentaram variações e na análise do conjunto das amostras "Com risco de queda" a variação ocorreu de acordo com algoritmo, com bons valores de acurácia. O objetivo é encontrar um ponto de corte que torne o sistema de detecção mais confiável para identificar situações de risco. Porém, a análise do desempenho dos detectores usando diferentes situações de risco de queda (3 grupos distintos) indica que para o detector baseado em $\mathrm{kNN}$ o ajuste do ponto de corte ótimo (que determina as situações de anomalia) varia menos que para os outros detectores. Futuramente, pretende-se testar a proposta em campo na enfermaria de um hospital.

\section{REFERÊNCIAS}

[1] Shih-Hau Fang, Chu-Chen Li, Wen-Chen Lu, Zhezhuang Xu, and Ying-Ren Chien. Enhanced device-free human detection: Efficient learning from phase and amplitude of channel state information. IEEE Transactions on Vehicular Technology, 68(3):3048-3051, 2019.

[2] Bruno Soares da Silva, Gustavo Teodoro Laureano, and Kleber Vieira Cardoso. Widmove - um sensor de movimento direcional baseado em perturbações do sinal eletromagnético de interfaces 802.11. In Anais do XXXVI Simpósio Brasileiro de Redes de Computadores e Sistemas Distribuídos. SBC, 2018.

[3] Jianfei Yang, Han Zou, Hao Jiang, and Lihua Xie. Devicefree occupant activity sensing using wifi-enabled IoT devices for smart homes. IEEE Internet of Things Journal, 5(5):3991-4002, 2018.

[4] Wei Wang, Alex X Liu, Muhammad Shahzad, Kang Ling, and Sanglu Lu. Understanding and modeling of wifi signal based human activity recognition. In Proceedings of the 21st annual international conference on mobile computing and networking, pages 65-76, 2015.

[5] Diana Yacchirema, Jara Suárez de Puga, Carlos Palau, and Manuel Esteve. Fall detection system for elderly people using IoT and big data. Procedia computer science, 130:603-610, 2018.

[6] Paulo Victor GF Dias, Edgar Douglas M Costa, Michel Pompeu Tcheou, and Lisandro Lovisolo. Fall detection monitoring system with position detection for elderly at indoor environments under supervision. In 2016 8th IEEE Latin-American Conference on Communications (LATINCOM), pages 1-6. IEEE, 2016.

[7] Andreas F Molisch. Wireless communications, volume 34. John Wiley \& Sons, 2012.

[8] V Djaja-Josko. Presence and fall detection in confined indoor environments using multiple uwb transceivers. In 2018 26th Telecommunications Forum (TELFOR), pages 1-4. IEEE, 2018.

[9] Jiang Xiao, Kaishun Wu, Youwen Yi, Lu Wang, and Lionel M Ni. Pilot: Passive device-free indoor localization using channel state information. In 2013 IEEE 33rd International Conference on Distributed Computing Systems, pages 236-245. IEEE, 2013.

[10] Kaishun Wu, Jiang Xiao, Youwen Yi, Dihu Chen, Xiaonan Luo, and Lionel $\mathrm{M}$ Ni. Csi-based indoor localization. IEEE Transactions on Parallel and Distributed Systems, 24(7):13001309, 2012
[11] Lutero Marques Oliveira. Câmeras filmadoras de monitoramento dentro e fora da unidade hospitalar. Arquivos do CRMPR, 29(115), 2012.

[12] José Albertino Souza. Vedada instalação de câmeras filmadoras nas salas de atendimento em serviços de emergência. Arquivos do CRM-PR, 33(130), 2016.

[13] Bo Tan, Karl Woodbridge, and Kevin Chetty. A wireless passive radar system for real-time through-wall movement detection. IEEE Transactions on Aerospace and Electronic Systems, 52(5):2596-2603, 2016.

[14] Bo Tan, Alison Burrows, Robert Piechocki, Ian Craddock, Qingchao Chen, Karl Woodbridge, and Kevin Chetty. Wi-fi based passive human motion sensing for in-home healthcare applications. In 2015 IEEE 2nd World Forum on Internet of Things (WF-IoT), pages 609-614. IEEE, 2015.

[15] Kun Qian, Chenshu Wu, Yi Zhang, Guidong Zhang, Zheng Yang, and Yunhao Liu. Widar2. 0: Passive human tracking with a single wi-fi link. In Proceedings of the 16th Annual International Conference on Mobile Systems, Applications, and Services, pages 350-361, 2018.

[16] Wenda Li, Bo Tan, and Robert J Piechocki. Wifi-based passive sensing system for human presence and activity event classification. IET Wireless Sensor Systems, 8(6):276-283, 2018.

[17] Wenda Li, Robert J Piechocki, Karl Woodbridge, Chong Tang, and Kevin Chetty. Passive wifi radar for human sensing using a stand-alone access point. IEEE Transactions on Geoscience and Remote Sensing, 2020.

[18] Espressif. Wi-fi driver. Disponível em: https://docs.espressif.com/projects/esp-idf/en/latest/esp32/ api-guides/wifi.html. Acesso em: 21 de março de 2021.

[19] Jerome Friedman, Trevor Hastie, Robert Tibshirani, et al. The elements of statistical learning, volume 1 . Springer series in statistics New York, 2001.

[20] Steven M Holland. Principal components analysis (PCA). Department of Geology, University of Georgia, Athens, GA, pages 30602-2501, 2008.

[21] Ankur A Patel. Hands-on unsupervised learning using Python: how to build applied machine learning solutions from unlabeled data. O'Reilly Media, 2019.

[22] TensorFlow Tutoriais. Introdução aos codificadores automáticos - Terceiro exemplo: detecção de anomalias. Disponível em: https://www.tensorflow.org/tutorials/generative/autoencoder. Acesso em: 03 de abril de 2021.

[23] Mariana Vitória de Menezes Bordalo Cristiano. Sensibilidade e especificidade na curva ROC: um caso de estudo. PhD thesis, Escola Superior de Tecnologia e Gestão, 2017.

[24] T. Fawcett. An introduction to ROC analysis. Pattern recognition letters, 27(8):861-874, 2006.

[25] Edson Zangiacomi Martinez, Francisco Louzada-Neto, and Basílio de Bragança Pereira. A curva ROC para testes diagnósticos. Cad. saúde colet.,(Rio J.), pages 7-31, 2003. 